TITLE:

\title{
Dynamics of optical gain in InxGa1- xN multi-quantum-well-based laser diodes
}

\section{$\operatorname{AUTHOR}(S)$ :}

Kawakami, Y; Narukawa, Y; Omae, K; Fujita, S; Nakamura, $\mathrm{S}$

\section{CITATION:}

Kawakami, Y ...[et al]. Dynamics of optical gain in InxGa1-xN multiquantum-well-based laser diodes. APPLIED PHYSICS LETTERS 2000, 77(14): 2151-2153

\section{ISSUE DATE:}

2000-10-02

URL:

http://hdl.handle.net/2433/50145

\section{RIGHT:}

Copyright 2000 American Institute of Physics. This article may be downloaded for personal use only. Any other use requires prior permission of the author and the American Institute of Physics. 


\title{
Dynamics of optical gain in $\ln _{x} \mathrm{Ga}_{1-x} \mathrm{~N}$ multi-quantum-well-based laser diodes
}

\author{
Yoichi Kawakami, ${ }^{\text {a) }}$ Yukio Narukawa, Kunimichi Omae, and Shigeo Fujita \\ Department of Electronic Science and Engineering, Kyoto University, Kyoto 606-8501, Japan \\ Shuji Nakamura ${ }^{\text {b) }}$ \\ Department of Research and Development, Nichia Chemical Industries Ltd., 491 Oka, Kaminaka, Anan, \\ Tokushima 774-8601, Japan
}

(Received 30 May 2000; accepted for publication 8 August 2000)

\begin{abstract}
Dynamical behavior of optical gain formation has been assessed at room temperature in the $\mathrm{In}_{x} \mathrm{Ga}_{1-x} \mathrm{~N}$ multi-quantum-well (MQW) based laser diodes (LDs) by employing pump and probe spectroscopy with a pulse width of 150 fs. The LDs are composed of (a) $\mathrm{In}_{0.1} \mathrm{Ga}_{0.9} \mathrm{~N}-\mathrm{In}_{0.02} \mathrm{Ga}_{0.98} \mathrm{~N}$ MQW and (b) $\mathrm{In}_{0.3} \mathrm{Ga}_{0.7} \mathrm{~N}-\mathrm{In}_{0.05} \mathrm{Ga}_{0.95} \mathrm{~N}$ MQW, whose stimulated emissions correspond to near ultraviolet $(390 \mathrm{~nm})$ and blue $(440 \mathrm{~nm})$, respectively. The optical gain was contributed from the nearly delocalized states [the lowest-quantized MQW levels (LQL)] in the sample (a), while it was from highly localized levels with respect to the LQL by $500 \mathrm{meV}$ for the sample (b). It was found that the photogenerated carriers rapidly (less than $1 \mathrm{ps)} \mathrm{transferred} \mathrm{to} \mathrm{the} \mathrm{LQL,} \mathrm{and} \mathrm{then} \mathrm{relaxed} \mathrm{to}$ the localized tail within the time scale of about $5 \mathrm{ps}$, giving rise to the optical gain. Such gain spectra were saturated and other bands appeared in the vicinity of the LQL under higher photoexcitation.

(C) 2000 American Institute of Physics. [S0003-6951(00)03240-X]
\end{abstract}

Recent development of the fabrication technology has opened the way to the commercialization of violet laser diodes (LDs), ${ }^{1,2}$ which are composed of InGaN/GaN/AlGaNbased heterostructures. The tuning wavelength for the stable continuous-wave (cw) operation of LDs is currently in the range between $376 \mathrm{~nm}$ (Ref. 3) and $450 \mathrm{~nm},{ }^{4}$ which is much narrower than that of light-emitting diodes due to dramatic increase of $I_{\text {th }}$ with increasing emission wavelength from 420 to $450 \mathrm{~nm}$. This may be because the internal electric field is so large in In-rich active layers that oscillator strength between electron and hole is suppressed, and/or because the optical gain cannot be generated sufficiently due to the inhomogeneous broadening effect resulting in the limited number of density of states. Further breakthrough is, therefore, required to realize pure blue and green LDs using InGaNbased semiconductors. Such targets can be facilitated by the well understanding of the emission mechanism, as well as by the well designing of LD structures.

So far, the assessment of stimulated emission has been reported in InGaN multi-quantum-well (MQW) structures. ${ }^{5-10}$ However, the mechanism is still controversial, where some groups claim that carrier localization plays a key role in both spontaneous and stimulated emissions, ${ }^{5-7}$ while another one reports that localized sates are saturated and delocalized states of electron-hole pairs contribute to the optical gain. ${ }^{8}$ Such discrepancy is probably because the degree of localization in InGaN active layers differs according to both the mean In-mole fraction and growth conditions, resulting in the difficulty in the general discussion on optical properties.

In this letter, the dynamics of gain formation is studied

\footnotetext{
${ }^{a)}$ Electronic mail: kawakami@kuee.kyoto-u.ac.jp

${ }^{b}$ Present address: Materials Department, University of California, Santa Barbara, CA 93106.
}

in a series of InGaN MQW LDs whose wavelength of stimulated emissions corresponds to near ultraviolet $(390 \mathrm{~nm})$ and blue $(440 \mathrm{~nm})$ by employing pump and probe spectroscopy.

Two types of LD structures, whose In content in the active layers are roughly (a) $10 \%$ and (b) $30 \%$ are assessed in this study. ${ }^{4,6,11-13}$ Whole samples ${ }^{14}$ consist of a separate confinement heterostructure where (a) a Si-doped $\mathrm{In}_{0.1} \mathrm{Ga}_{0.9} \mathrm{~N}$ $(3 \mathrm{~nm}) / \mathrm{In}_{0.02} \mathrm{Ga}_{0.98} \mathrm{~N}(6 \mathrm{~nm}) \mathrm{MQW}$ with three periods and (b) an undoped $\mathrm{In}_{0.3} \mathrm{Ga}_{0.7} \mathrm{~N}(3 \mathrm{~nm}) / \mathrm{In}_{0.05} \mathrm{Ga}_{0.95} \mathrm{~N}(10 \mathrm{~nm}) \mathrm{MQW}$ with six periods are sandwiched between $\mathrm{GaN}$ waveguide layers $\left(0.1 \mu \mathrm{m}\right.$ in each) and $\mathrm{Al}_{0.15} \mathrm{Ga}_{0.85} \mathrm{~N}$ cladding layers (0.4 $\mu \mathrm{m}$ in each). The top of the $\mathrm{Al}_{x} \mathrm{Ga}_{1-x} \mathrm{~N}$ clad and the $\mathrm{GaN}$ waveguide are $\mathrm{Mg}$-doped $p$-type layers, while the bottom of the clad and the waveguide are Si-doped $n$-type layers. The LD operation at room temperature (RT) was performed at $390 \mathrm{~nm}$ under $\mathrm{cw}$ mode for sample (a) (near-UV $\mathrm{LD}$ ) and at $440 \mathrm{~nm}$ under pulsed mode for sample (b) (blue LD).

The pump and probe spectroscopy depicted was performed for the measurement of temporal behavior of differential absorption using a dual photodiode array in conjunction with a $25 \mathrm{~cm}$ monochromator. ${ }^{15}$ The white light used for the probe beam was generated by focusing part of the output beam from the regenerative amplifier on a $\mathrm{D}_{2} \mathrm{O}$ cell. The pulse width of both the pump and probe beam was $150 \mathrm{fs}$. Delay time of the probe beam with respect to the pump beam was tuned by changing the position of the retroreflector which could be controlled by the pulse stage. In order to detect the probe beam with spatially uniform carrier distribution in the sample, the focus size of the pump beam $(600$ $\mu \mathrm{m}$ in diameter) was set so as to be much larger than that of the probe beam (200 $\mu \mathrm{m}$ in diameter). Furthermore, the probe beam was perpendicularly polarized with respect to the pump beam, and the transmitted probe beam polarized in this direction was detected to avoid the scattered component 

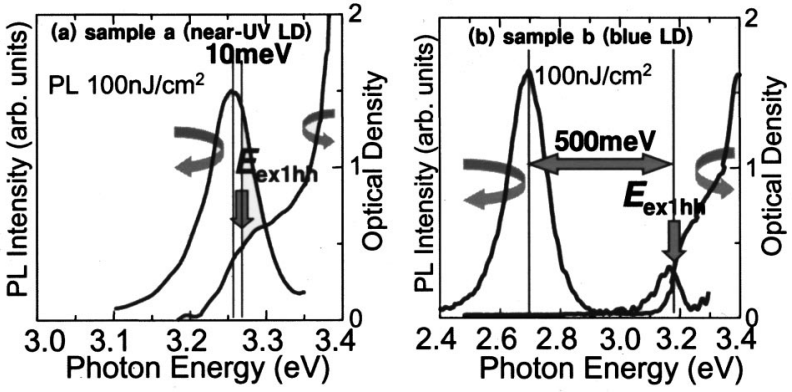

FIG. 1. Time-integrated PL under $I_{\mathrm{ex}}=100 \mathrm{~nJ} / \mathrm{cm}^{2}$ and optical density spectra under low photoexcitation energy density taken at (a) sample (a) $\left(\mathrm{In}_{0.1} \mathrm{Ga}_{0.9} \mathrm{~N} / \mathrm{In}_{0.02} \mathrm{Ga}_{0.98} \mathrm{~N}-\mathrm{MQW}\right.$ near-UV LD) and (b) sample (b) $\left(\mathrm{In}_{0.3} \mathrm{Ga}_{0.7} \mathrm{~N} / \mathrm{In}_{0.05} \mathrm{Ga}_{0.95} \mathrm{~N}-\mathrm{MQW}\right.$ blue LD).

of the pump beam. The whole measurements have been done at RT.

In the pump and probe spectroscopy, the transmission spectrum of the probe beam detected in the presence of the pump beam $(T+\Delta T)$ is compared to the spectrum without the pump beam $(T)$, giving the frequency $(\omega)$-dependent $\Delta T\left(\omega, I_{\mathrm{ex}}, t_{d}\right)$ of the sample for different intensities of the pump $\left(I_{\mathrm{ex}}\right)$, and for different time delays after the pulsed pumping $\left(t_{d}\right)$. The photoinduced change of optical density $\left[\Delta \mathrm{OD}\left(\omega, I_{\mathrm{ex}}, t_{d}\right)\right]$,

$$
\Delta \mathrm{OD}=\log \left(\frac{T}{T+\Delta T}\right)=\Delta \alpha d \times 0.434,
$$

where $\Delta \alpha\left(\omega, I_{\mathrm{ex}}, t_{d}\right)$ is the photoinduced change of absorption coefficient and $d$ is the thickness of absorbing layer.

Figure 1 shows time-integrated photoluminescence (TIPL) spectra under photoexcitation energy density $\left(I_{\mathrm{ex}}\right)$ of $100 \mathrm{~nJ} / \mathrm{cm}^{2}$ taken at two types of LDs. Absorption spectra under low photoexcitation (Xe lamp) corresponding to the density of states are also depicted in Fig. 1. The lowest quantized energy levels within the wells (designated as $E_{\text {ex1hh }}$ ) were estimated by electroreflectance spectroscopy. In contrast to sample (a), the PL of sample (b) is composed of two emission bands, and the main bands are located on the lowenergy side with respect to $E_{\text {exlhh }}$ by $500 \mathrm{meV}$. Blueshift of
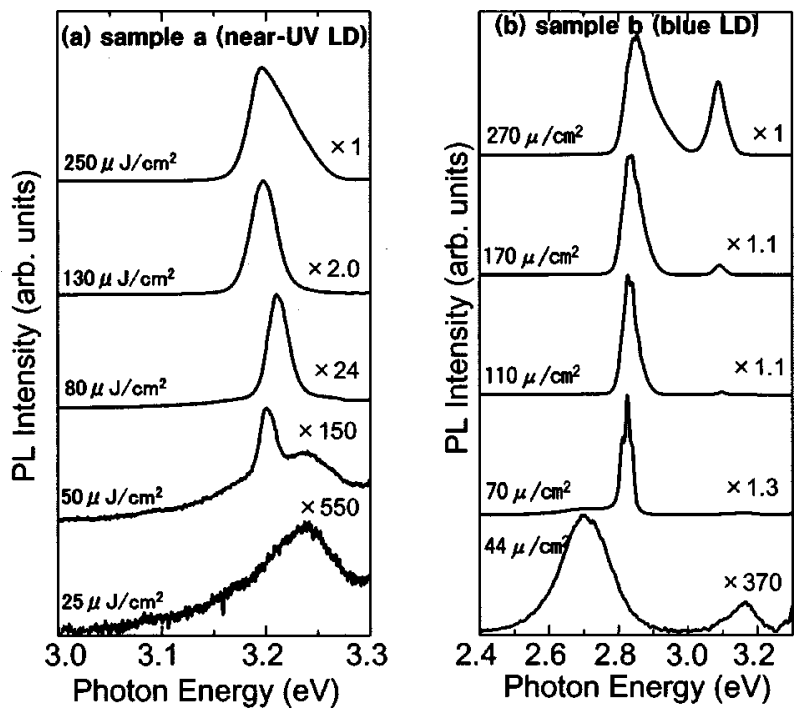

FIG. 2. Time-integrated PL from the resonator as a function of excitation

FIG. 2. Time-integrated PL from the resonator as a function of excitation
energy density taken at (a) sample (a) and (b) sample (b). Downloaded 04 Mar 2008 to 130.54.110.22. Redistribution subject
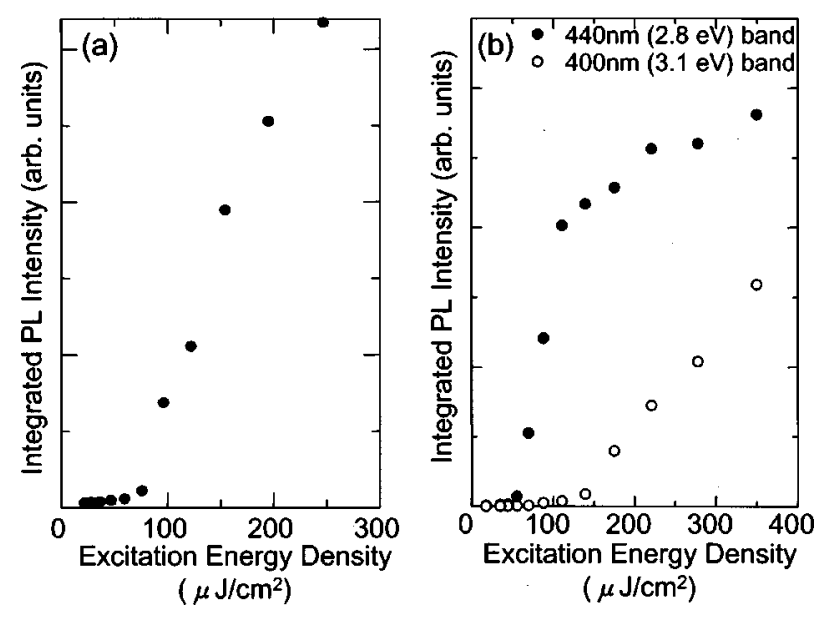

FIG. 3. Integrated PL intensity for the emission bands as a function of excitation energy density taken at (a) sample (a) and (b) sample (b).

these bands under increased $I_{\mathrm{ex}}$ conditions (from $10 \mathrm{~nJ} / \mathrm{cm}^{2}$ to $20 \mu \mathrm{J} / \mathrm{cm}^{2}$ ) is about a few tens $\mathrm{meV}$, which is substantially smaller that the energy difference. These phenomena were attributed to the localization of excitons whose origin is probably due to In-rich quantum dot (QD) like centers. This model is supported by the energy-dispersive $\mathrm{x}$-ray microanalysis using scanning transmission electron microscopy. ${ }^{5}$ Moreover, the zero-dimensional feature of excitons is revealed especially in blue-emitting quantum wells (QWs) where almost no temperature dependence of the radiative lifetime was observed from cryogenic temperature to RT. ${ }^{13}$

The TI-PL spectra were detected with the edge geometry from the resonator cavity-the length of which was about 1 mm. As shown in Figs. 2 and 3, stimulated emission (SE) was observed from every sample if $I_{\mathrm{ex}}$ is more than about $50 \mu \mathrm{J} / \mathrm{cm}^{2}$. SE appears on the low-energy side of the spontaneous emission (SPE) by $32 \mathrm{meV}$ for sample (a), while from the high-energy side of the main SPE band by $130 \mathrm{meV}$ for sample (b). The Large blueshift in sample (b) may be due to the band-filling effects within localized states, and/or due to the screening effect of the internal electric field. Nevertheless, this energy is still substantially smaller than the localization energy (500 meV) observed in the SPE. Therefore, it is probable that the optical gain was contributed from the localized centers for the blue LD mean-In composition of which in the wells is about $30 \%$. However, as shown in Fig. 3(b), these SE bands are saturated if $I_{\mathrm{ex}}$ is more than about $100 \mu \mathrm{J} / \mathrm{cm}^{2}$, then new SE bands grow on the low-energy side of the SPE bands originating from the $E_{\text {exlhh }}$ transition.

The dynamical behavior of dense carriers was characterized by measurement of the differential absorption coefficient by employing pump and probe spectroscopy. Figure 4(a) shows the variation of differential optical density spectra as a function of time after pumping at $370 \mathrm{~nm}$ under $I_{\mathrm{ex}}$ $=200 \mu \mathrm{J} / \mathrm{cm}^{2}$ in sample (a) $\left(\mathrm{In}_{0.1} \mathrm{Ga}_{0.9} \mathrm{~N}\right.$ QW, near-UV LD). The optical density spectrum taken under the low $I_{\mathrm{ex}}$ condition is depicted as a reference. PL spectra of both SE $\left(I_{\mathrm{ex}}\right.$ $\left.=200 \mu \mathrm{J} / \mathrm{cm}^{2}\right)$ and SPE $\left(I_{\mathrm{ex}}=100 \mathrm{~nJ} / \mathrm{cm}^{2}\right)$ taken from the surface are also shown in Fig. 4(a). The sheet carrier density is roughly estimated to be $1.9 \times 10^{13} \mathrm{~cm}^{-2}$ under $I_{\mathrm{ex}}$ $=200 \mu \mathrm{J} / \mathrm{cm}^{2}$. At the initial stage of excitation $(t=$
AIP license or copyright; see http://apl.aip.org/apl/copyright.jsp 

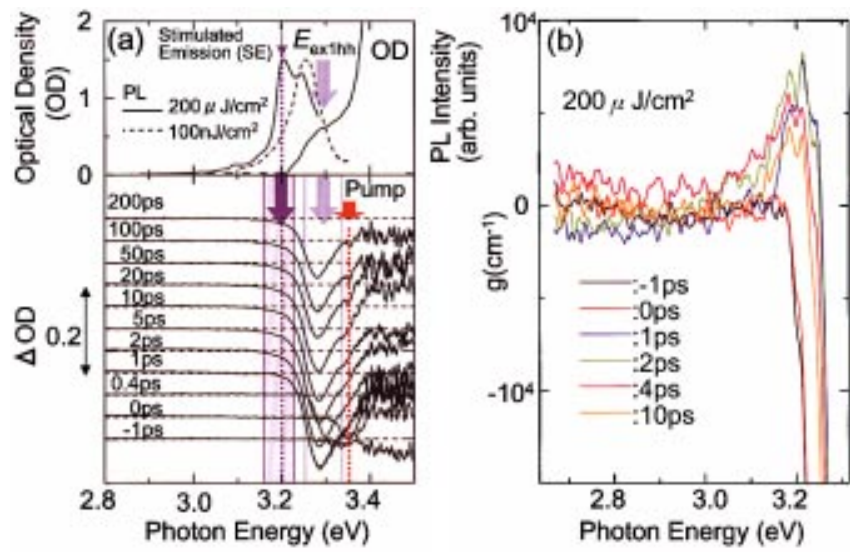

FIG. 4. (Color) (a) Differential optical density spectra as a function of time delay under $I_{\mathrm{ex}}=200 \mu \mathrm{J} / \mathrm{cm}^{2}$ taken at sample (a) (near-UV LD). Optical density (OD) spectrum taken under low photoexcitation energy density, as well as PL spectra taken from the surface under $I_{\mathrm{ex}}=100 \mathrm{~nJ} / \mathrm{cm}^{2}$ [spontaneous emission (SPE)] and 200 under $I_{\mathrm{ex}}=200 \mu \mathrm{J} / \mathrm{cm}^{2}$ [stimulated emission (SE)] are depicted in the figure. (b) Dynamics of optical gain evaluated by the data in (a).

$-0.5-0 \mathrm{ps}$ ), bleaching of the photoabsorption was observed in the vicinity of the pumping energy. However, the negative peak of $\Delta \mathrm{OD}$ rapidly reached the level of $E_{\text {exihh }}$ at a time scale of less than $1 \mathrm{ps}$. The red shift of $E_{\text {ex } 1 \mathrm{hh}}$ was observed with increasing time $(13 \mathrm{meV}$ at $t=200 \mathrm{ps})$, probably because screening of the piezoelectric field is reduced with decreasing carrier density. ${ }^{16}$ Optical gain was generated on the low-energy side of the negative peak due to the effect of band-gap renormalization. As shown in Fig. 4(b), maximum optical gain as large as about $8000 \mathrm{~cm}^{-1}$ was reached at $t$ $=2 \mathrm{ps}$.

The dynamics of dense carriers was also assessed for sample (b) (blue LD), as shown in Fig. 5(a). The tail of differential absorption began to grow toward the bottom of the localized levels just after the bleaching at $E_{\text {exlhh }}$ is completed. The time scale for reaching to the bottom was about 5 ps. Therefore, it was found that photogenerated carriers and/or excitons efficiently transfer to deeply localized levels without the effect of a phonon bottleneck observed in other QD systems. As described before, stimulated emission originating from localized centers [designated as $\mathrm{SE}_{1}$ in Fig. 5(a)] saturates under higher photoexcitation, and a new SE from $E_{\text {exlhh }}\left(\mathrm{SE}_{2}\right)$ appears in these samples. Figure 5(b) shows the dynamics of optical gain spectra under $I_{\mathrm{ex}}=200 \mu \mathrm{J} / \mathrm{cm}^{2}$ taken at sample (b) (blue LD). $\mathrm{SE}_{2}$ began to grow at $t$ $=1-2 \mathrm{ps}$ and reached the maximum $\left(g=3000 \mathrm{~cm}^{-1}\right)$ at $t$ $=4 \mathrm{ps}$, while the temporal behavior of $\mathrm{SE}_{1}$ is delayed by about 2 ps with respect to that of $\mathrm{SE}_{2}$.

In conclusion, the dynamics of dense carriers has been studied at RT in (a) $\mathrm{In}_{0.1} \mathrm{Ga}_{0.9} \mathrm{~N} / \mathrm{In}_{0.02} \mathrm{Ga}_{0.98} \mathrm{~N}$ and (b) $\mathrm{In}_{0.3} \mathrm{Ga}_{0.7} \mathrm{~N} / \mathrm{In}_{0.05} \mathrm{Ga}_{0.95} \mathrm{~N}$ MQW LD structures by means of pump and probe spectroscopy. The optical gain was contributed from the nearly delocalized state of the QW ( $\left.E_{\text {exilhh }}\right)$ in sample (a). However, it was generated from both QD-like localized centers and the QW under moderately high photoexcitation in sample (b). It was found that the photogener-

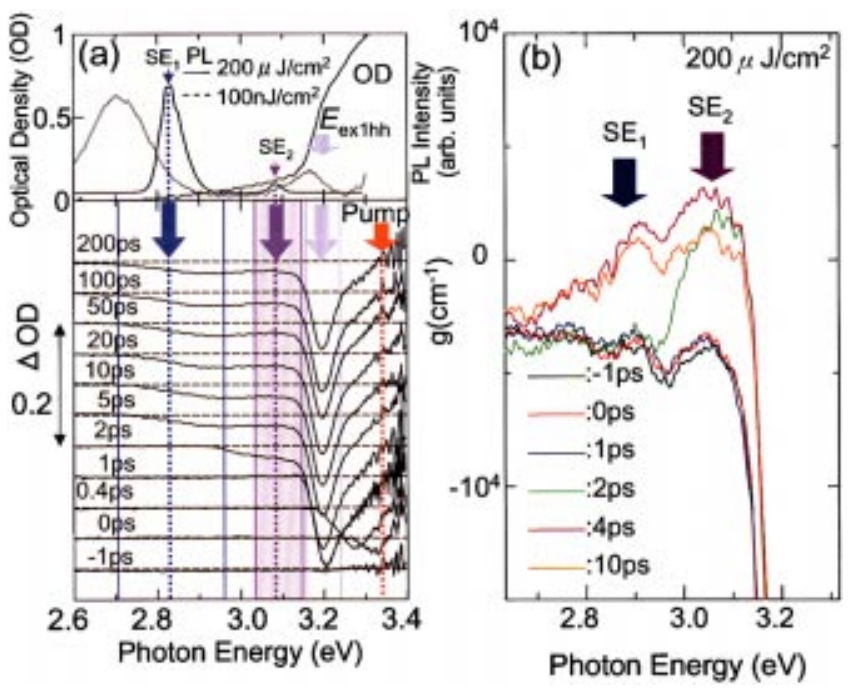

FIG. 5. (Color) (a) Differential optical density spectra as a function of time delay under $I_{\mathrm{ex}}=200 \mu \mathrm{J} / \mathrm{cm}^{2}$ taken at sample (b) (blue LD). OD spectrum, SPE spectrum under $I_{\mathrm{ex}}=100 \mathrm{~nJ} / \mathrm{cm}^{2}$, and SE spectrum under $I_{\mathrm{ex}}$ $=200 \mu \mathrm{J} / \mathrm{cm}^{2}$ are depicted in the figure. Two SE bands designated as $\mathrm{SE}_{1}$ and $\mathrm{SE}_{2}$ are also observed for the detection from the surface. (b) Dynamics of optical gain evaluated by the data in (a).

ated carriers rapidly (less than $1 \mathrm{ps)}$ transferred to the LQL, and then relaxed to the localized tail within the time scale of several ps, giving rise to the optical gain.

The authors are grateful for the financial support of the Grant-in-Aid for Scientific Research from the Ministry of Education, Science, Sports and Culture. A part of this work was performed using the facility at the Venture Business Laboratory in Kyoto University (KU-VBL).

${ }^{1}$ S. Nakamura, M. Senoh, S. Nagahama, N. Iwasa, T. Yamada, T. Matsushita, H. Kiyoku, and Y. Sugimoto, Jpn. J. Appl. Phys., Part 2 35, L74 (1996).

${ }^{2}$ S. Nakamura, M. Senoh, S. Nagahama, T. Matsushita, H. Kiyoku, Y. Sugimoto, T. Kozaki, H. Umemoto, M. Sano, and T. Mukai, Jpn. J. Appl. Phys., Part 2 38, L226 (1999).

${ }^{3}$ I. Akasaki, H. Amano, S. Sota, H. Sakai, T. Tanaka, and M. Koike, Electron. Lett. 32, 1105 (1996).

${ }^{4}$ S. Nakamura, M. Senoh, S. Nagahama, N. Iwasa, T. Matsushita, and T. Mukai, Appl. Phys. Lett. 76, 22 (2000).

${ }^{5}$ Y. Narukawa, Y. Kawakami, M. Funato, Sz. Fujita, Sg. Fujita, and S. Nakamura, Appl. Phys. Lett. 70, 868 (1997).

${ }^{6}$ Y. Narukawa, Y. Kawakami, Sg. Fujita, and S. Nakamura, Phys. Status Solidi A 176, 39 (1999).

${ }^{7}$ T. Deguchi, T. Azuhata, T. Sota, S. Chichibu, and S. Nakamura, Mater. Sci. Eng., B 50, 251 (1997).

${ }^{8}$ G. Mohs, T. Aoki, M. Nagai, R. Shimano, M. Kuwata-Gonokami, and S. Nakamura, Solid State Commun. 104, 643 (1997).

${ }^{9}$ A. Satake, Y. Masumoto, T. Miyajima, T. Asatsuma, and M. Ikeda, Phys. Rev. B 60, 16660 (1999).

${ }^{10}$ Y. H. Cho, T. J. Schmidt, S. Bidnyk, G. H. Gainer, J. J. Song, S. Keller, U. K. Mishra, and S. P. DenBaars, Phys. Rev. B 61, 7571 (2000).

${ }^{11}$ Y. Narukawa, Y. Kawakami, Sz. Fujita, Sg. Fujita, and S. Nakamura, Phys. Rev. B 55, R1938 (1997).

${ }^{12}$ Y. Narukawa, Y. Kawakami, Sg. Fujita, and S. Nakamura, Phys. Rev. B 59, 10283 (1999).

${ }^{13}$ Y. Kawakami, Y. Narukawa, K. Omae, Sg. Fujita, and S. Nakamura, Phys. Status Solidi A 178, 331 (2000).

${ }^{14}$ S. Nakamura, Jpn. J. Appl. Phys., Part 2 30, L1705 (1991).

${ }^{15}$ Y. Narukawa, Ph.D. thesis, Kyoto University (2000).

${ }^{16}$ T. Takeuchi, S. Sota, M. Katsuragawa, M. Komori, H. Takeuchi, H. Amano, and I. Akasaki, Jpn. J. Appl. Phys., Part 2 36, L382 (1997). 